\title{
Recent Topics on Very High Energy Gamma-ray Astronomy
}

\author{
Masaki MorI* \\ Institute for Cosmic Ray Research, University of Tokyo \\ 5-1-5 Kashiwanoha, Kashiwa, Chiba 277-8582, Japan
}

\begin{abstract}
With the advent of imaging atmospheric Cherenkov telescopes in late 1980's, ground-based observation of $\mathrm{TeV}$ gamma-rays came into reality after struggling trials by pioneers for twenty years, and the number of gamma-ray sources detected at $\mathrm{TeV}$ energies has increased to be over seventy now. In this review, recent findings from ground-based very-high-energy gamma-ray observations are summarized (as of $2008 \mathrm{March}$ ), and up-to-date problems in this research field are presented.
\end{abstract}

KEYWORDS: gamma-rays, supernova remnants, pulsar nebulae, active galactic nuclei

\section{Introduction}

There has been a dramatic progress in very-highenergy $^{1)}$ gamma-ray astronomy in the last decade. With the advent of imaging atmospheric Cherenkov telescopes, the number of gamma-ray sources detected in the $\mathrm{TeV}$ energy range has increased significantly in the last several years, and now it exceeds seventy, according to the rapporteur talk by Jim Hinton at the International Cosmic Ray Conference in 2007. ${ }^{2}$ ) These gamma-ray sources form a new class of high-energy objects in the Universe, including active galactic nuclei, radio galaxies, galactic binaries, pulsar wind nebulae, in addition to supernova remnants which are assumed to be the origin of cosmicrays for a long time. Exploring the emission mechanism from these objects is a big challenge in astrophysics. Non-thermal nature of emission inherently needs multiwavelength observations to study the phenomena, involving astronomers working in other wavelength. Furthermore, many unidentified gamma-ray sources have been reported and are posing new mysteries.

In this review, highlights of recent findings from ground-based very-high-energy gamma-ray observations are summarized, and up-to-date problems in this research field are presented. We concentrate on recent results from Cherenkov telescopes here, but new results from particle shower arrays are also exciting (G. Sinnis and M. Takita, in these proceedings.) Recent review on this field is also found elsewhere. ${ }^{3)}$

\section{Imaging Atmospheric Cherenkov Telescopes}

Ground-based imaging Cherenkov telescopes are becoming a powerful tool to study very high energy gammarays with their capability to discriminate gamma-rays from background hadrons (protons and nuclei). ${ }^{4)}$

Gamma-ray images come from purely electromagnetic showers and are sharp and oriented toward the object being tracked. They can be separated from cosmicray showers using imaging parameters first defined by A.M. Hillas: ${ }^{5)}$ width, length, distance, asymmetry. Distributions of theses parameters for gamma-ray showers are different from those for hadronic showers, and we can extract gamma-ray signals statistically from observed im- ages. The first firm detection of a $\mathrm{TeV}$ gamma-ray signal from the Crab nebula by the Whipple group ${ }^{6)}$ utilized this imaging technique.

Stereoscopic observation of Cherenkov images came into practical use by the HEGRA group. ${ }^{7)}$ Incoming direction of a gamma-ray can be determined by intersection of axes of elongated Cherenkov images observed by multiple telescopes, separated by about $100 \mathrm{~m}$, which is the size of light pool of Cherenkov light flash, more precisely compared to single telescope observation. Distance of centroid of shower images from the incoming direction is a measure of shower maximum height in the atmosphere, which also allows better estimation of gammaray energy. In addition, background Cherenkov flashes caused by local cosmic-ray muons traversing near telescopes can be effectively rejected requiring coincidence between telescopes.

Table I summarizes characteristics of Cherenkov telescope systems in operation.

\section{Recent Topics}

Table II is a summary of TeV sources which are fairly established (detected by multiple groups and/or detected at high significance). Over 40 sources in this table were found by the H.E.S.S. group, mostly by their Galactic plane survey. ${ }^{8)}$ Note the classification of sources are not unique and depend on person who makes a list: identification of $\mathrm{TeV}$ sources with objects found in other wavelength are sometimes difficult, because of the limited angular resolution of $\mathrm{TeV}$ observation: therefore many $\mathrm{TeV}$ sources are left unidentified. (But there are 'dark' sources: see section 3.1.6.)

Fig. 1 is a skymap of $\mathrm{TeV}$ sources in the Galactic coordinates compiled by R. Wagner. ${ }^{10)}$ Concentration of sources along the Galactic plane is obvious, but please note the exposure time is far from uniform.

\subsection{Galactic Sources \\ 3.1.1 Supernova Remnants}

Needless to say, supernova remnants (SNRs) are long considered to be primary sources for galactic cosmic rays. ${ }^{11)}$ They are energetic enough to support the cosmic ray luminosity of the Galaxy and their sizes are 
Table I. Current Cherenkov telescope systems

\begin{tabular}{|l||c|c|c|c|c|c|}
\hline Group & Location & Latitude & Longitude & Height & Telescopes & Start \\
\hline \hline SHALON & Russia & $42^{\circ} \mathrm{S}$ & $75^{\circ} \mathrm{E}$ & $3,338 \mathrm{~m}$ & $11 \mathrm{~m}^{2} \times 2$ & 1992 \\
\hline TACTIC & India & $25^{\circ} \mathrm{S}$ & $73^{\circ} \mathrm{E}$ & $1,300 \mathrm{~m}$ & $9.5 \mathrm{~m}^{2} \times 4$ & 2000 \\
\hline CANGAROO-III & Australia & $31^{\circ} \mathrm{S}$ & $137^{\circ} \mathrm{E}$ & $160 \mathrm{~m}$ & $57 \mathrm{~m}^{2} \times 4$ & 2004 \\
\hline H.E.S.S. & Namibia & $23^{\circ} \mathrm{S}$ & $16.5^{\circ} \mathrm{E}$ & $1,800 \mathrm{~m}$ & $107 \mathrm{~m}^{2} \times 4$ & 2004 \\
\hline MAGIC & Canary Is. & $29^{\circ} \mathrm{N}$ & $18^{\circ} \mathrm{W}$ & $2,200 \mathrm{~m}$ & $237 \mathrm{~m}^{2} \times 1$ & 2004 \\
\hline VERITAS & Arizona & $32^{\circ} \mathrm{N}$ & $111^{\circ} \mathrm{W}$ & $1,300 \mathrm{~m}$ & $110 \mathrm{~m}^{2} \times 4$ & 2007 \\
\hline
\end{tabular}

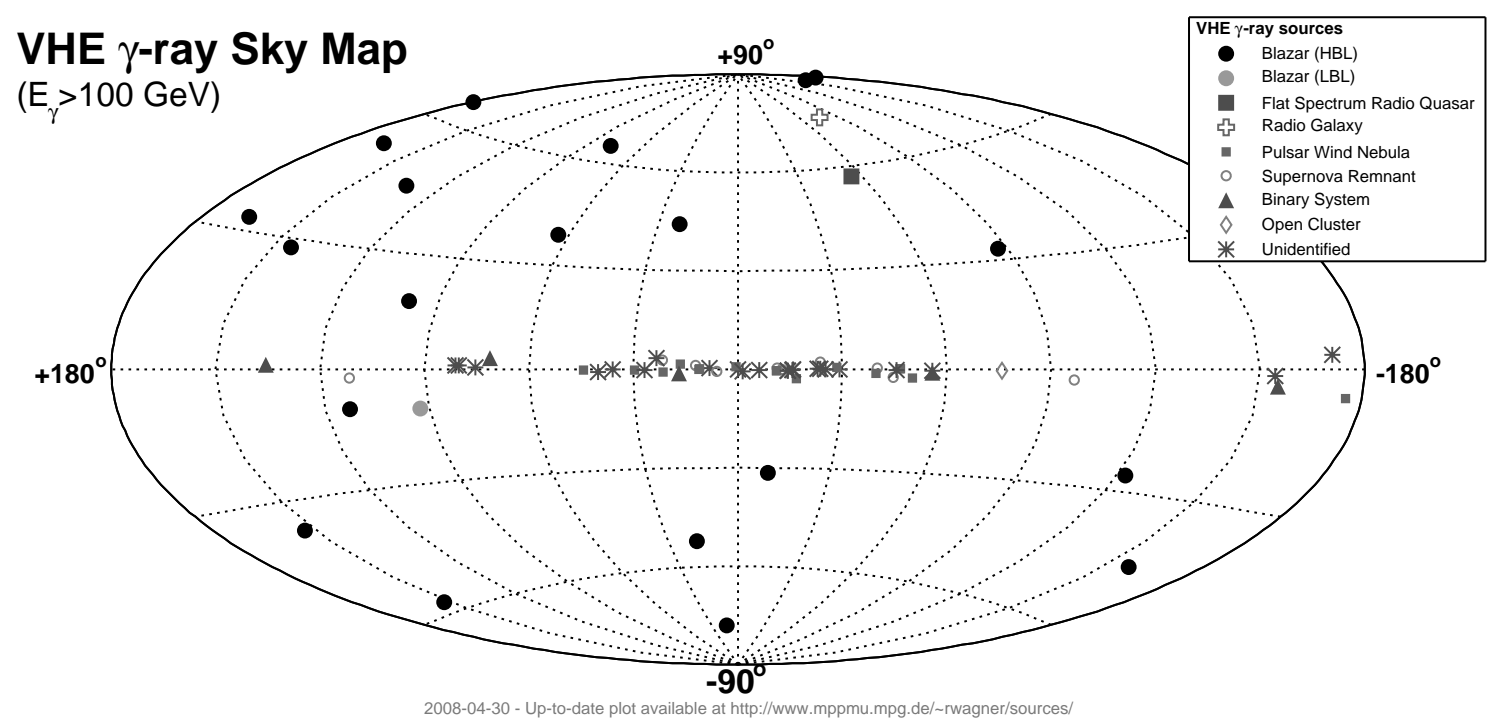

Fig. 1. TeV skymap (circa early 2008) compiled by R. Wagner. ${ }^{10)}$

Table II. Summary of TeV sources as of March 20089)

\begin{tabular}{|l|r|}
\hline Source type & Count \\
\hline \hline Galactic sources & 3 \\
Shell-type supernova remnant & 11 \\
Supernova remnant & 16 \\
Pulsar wind nebula & 4 \\
Binary & 1 \\
Galactic center & 1 \\
Galactic ridge & 1 \\
Young stellar cluster & 14 \\
Unidentified & 17 \\
Extragalactic sources & 1 \\
High-frequency peaked BL Lac (HBL) & 1 \\
Low-frequency peaked BL Lac (LBL) & 1 \\
Radio galaxy & 71 \\
Flat-spectrum radio quasar (FSRQ) & \\
\hline Total & \\
\hline
\end{tabular}

large enough to confine cosmic rays during acceleration by diffusive shockwave. Bright synchrotron radio emission from many SNRs indicate existence of non-thermal, high-energy electrons. Evidences of acceleration of protons in SNR, however, were rare.

The first SNR detected at $\mathrm{TeV}$ energies is $\mathbf{R X}$ J1713.7-3946 (by CANGAROO-I, ${ }^{12}$ ) CANGAROOII, ${ }^{13)}$ and H.E.S.S. ${ }^{14)}$ ) where the shell structure was clearly observed. ${ }^{14)}$ Although the spectrum is better explained by emission from neutral pions produced in hadronic interaction of protons, ${ }^{13-15)}$ inverse Compton emission by electrons is still a viable process to ac- count for the $\mathrm{TeV}$ spectrum. RX J0852.0-4622 (by CANGAROO-II ${ }^{16)}$ and H.E.S.S. ${ }^{17)}$ ) and RCW 86 (by H.E.S.S. ${ }^{18)}$ ) are other SNRs which show shell-like emission profile, well correlated with X-ray intensity profiles. Cas A, first claimed by HEGRA ${ }^{19)}$ and confirmed to be a TeV emitter by MAGIC, ${ }^{20)}$ and CTA 37A/37B, recently claimed by H.E.S.S. ${ }^{21)}$ could also be shell-like SNRs, but their small angular sizes do now allow further study. These shell-like structure are often well correlated with X-ray images, and one may suppose $\mathrm{TeV}$ emission comes from inverse Compton scattering of low energy photons by high-energy electrons which radiate synchrotron X-rays. ${ }^{22)}$ Still, gamma-rays derived from neutral pion decays produced in collision of high-energy protons with ambient matter could account for TeV emission, ${ }^{23)}$ since amplified magnetic fields in SNRs might suppress inverse Compton emission. ${ }^{24)}$

Uchiyama et al. analyzed the X-ray images of RX J1713.7-3946 observed by Chandra in multiple years, and found there are 'hot spots' showing time variation in oneyear scale. ${ }^{25)}$ This time scale, compared with the cooling time of high energy electrons, means the ambient magnetic field is stronger than $1 \mathrm{mG}$. This magnetic field suppresses the inverse Compton emission in the $\mathrm{TeV}$ region, and may indicate $\mathrm{TeV}$ emission is produced by hadronic process. However, Butt et al. argue that the existing multiwavelength data on this object do not support such conclusion. ${ }^{26)}$

On the other hand, TeV emission profiles of IC443 (by MAGIC $^{27)}$ and VERITAS ${ }^{28)}$ ) and W28 (by H.E.S.S. ${ }^{29)}$ ) 
Table III. TeV sources associated with pulsar wind nebulae. ${ }^{2,9}$ ) These associations were established through a range of methods, which are given in the table in abbreviated form: Pos: The position of the centroid of the VHE emission can be established with sufficient precision that there is no ambiguity as to the low energy counterpart. Mor: There is a match between the gamma-ray morphology and that seen at other (usually X-ray) wavelengths. EDMor: Energy-dependent morphology which approaches the position/morphology seen at other wavelengths at some limit, and is consistent with our physical understanding of the source.

\begin{tabular}{|l|l|c|l|}
\hline Object & Pulsar & Method & Discovered \\
\hline \hline Crab nebula & B0531+21 & Pos & Whipple, 1989 \\
MSH 15-52 & B1509-58 & Mor & HESS, 2005 \\
Vela X & B0833-45 & Mor & HESS, 2006 \\
HESS J1825-137 & B1823-13 & EDMor & HESS, 2005 \\
PSR J1420-6049 & J1420-6048 & Mor & HESS, 2006 \\
The Rabbit & J1420-6048 & Mor & HESS, 2006 \\
G0.9+0.1 & - & Pos & HESS, 2005 \\
\hline
\end{tabular}

do not show shell-like structure and seems to correlate with molecular cloud profiles observed by a $\mathrm{CO}$ emission line. This interaction may indicate the hadronic origin of gamma-rays produced in collisions of high-energy protons with molecular cloud as targets.

HESS J0632+057 was found close to the rim of the Monoceros Loop SNR/Rosette Nebula region. ${ }^{30)}$ It is point-like and has no clear counterpart at other wavelengths, but is possibly associated with the GeV source 3EG J0634+0521, a weak X-ray source 1RXS J063258.3+054857 and the Be-star MWC 148.

Thus, although supernova remnants are proved to be the sites producing high energy particles in the Universe, species of accelerated particles are still unclear: ${ }^{31)}$ solving the long-standing mystery of cosmic ray origin is still an important issue of gamma-ray astronomy.

\subsubsection{Pulsar Wind Nebulae}

Table III is a list of $\mathrm{TeV}$ sources with well established association with pulsar wind nebulae. Including associations with weaker evidence, 18 out of 71 sources detected at $\mathrm{TeV}$ energies seem to be associated with pulsar wind nebulae, ${ }^{2)}$ which was a rather surprising discovery revealed by the H.E.S.S. Galactic survey. ${ }^{8)}$ Most of them are associated with relatively young $\left(<10^{5}\right.$ years $)$ and large spin-down pulsars, ${ }^{32)}$ which means the gamma-ray luminosity is supported by the pulsar spin-down energy. Their profiles show extended (order of $10 \mathrm{pc}$ ) structure, often displaced from pulsar positions. No pulsation has been reported in the $\mathrm{TeV}$ region, even for the Crab nebula which is a strong $\mathrm{TeV}$ source and best studied by a number of groups. Thus the $\mathrm{TeV}$ emission are naturally ascribed to inverse Compton emission by highenergy electrons accelerated in the vicinity of pulsars.

This hypothesis on emission mechanism is further supported by the energy-dependent morphology in HESS J1825-137. ${ }^{33)}$ The photon indices from a power-law fit in different regions show a softening of the spectrum with increasing distance from the pulsar. The observed energy dependent morphology may be an evidence for cooling of electrons in the nebula.

HESS J1837-069, which was classified as unidenti- fied before, has now been added to this category with the discovery of an energetic $70.5 \mathrm{~ms}$ pulsar in $\mathrm{AX}$ J1838.0-0655 using X-ray data by RXTE. ${ }^{34)}$

\subsubsection{Gamma-ray Binaries}

PSR B1259-63/SS2883 This is a $48 \mathrm{~ms}$ pulsar and a Be star binary in a highly eccentric orbit. Gamma-ray flux at detectable level was predicted when the binary is near the periastron passage via interaction of pulsar wind with the radiative environment of the binary system. ${ }^{35)}$ H.E.S.S. detected such a variable flux around the 2004 periastron, ${ }^{36)}$ with a double-peak light curve as predicted by some models. ${ }^{37)}$

LS5039 This is a high mass X-ray binary comprising a massive star and a compact object, and is resolved into a bipolar radio outflow emanating from a central core, thus it is often classified as the microquasar class. H.E.S.S. detected a modulated gamma-ray signal with the 3.9 day orbital period in $2005 .{ }^{38)}$ The emission maximum $(\phi \sim 0.7)$ appears to lag behind the apastron epoch and to align better with the inferior conjunction $(\phi=0.716)$, when the compact object lies in front of the massive star. The flux minimum occurs at phase $(\phi \sim 0.2)$, slightly further along the orbit than superior conjunction $(\phi=0.058)$. This behavior is not easy to explain by a simple assumption and offers a challenge to model builders.

LSI+61 303 MAGIC observed this microquasar, known in the $\mathrm{GeV}$ region as $2 \mathrm{CG} 135+01$ (by COS B) or 3EG J0241+6103 (by EGRET), for six orbital cycles $\left(P_{\text {orb }}=26.5\right.$ days $)$ and obtained a modulated gamma-ray signal which is significant only between orbital phase 0.4 and $0.7{ }^{39)}$ The flux maximum is detected at phases $0.5^{-}$ 0.6 , overlapping with an X-ray outburst and the onset of a radio outburst, is shifted from the phase when the two stars are closest to one another, implying a strong orbital modulation of the emission or the absorption processes. The VERITAS group confirmed the modulated emission. ${ }^{40)}$

Cyg X-1 This well-known black hole X-ray binary was observed by MAGIC for a total of 40 hours during 26 nights in 2006. Although the steady TeV emission was not observed, a $4 \sigma$-level ( $3.2 \sigma$ after trial correction) evidence was obtained for one night on September 24, coinciding with an X-ray flare. ${ }^{41)}$ The significance of detection may not be strong enough to claim Cyg X-1 is a $\mathrm{TeV}$ gamma-ray source. ${ }^{2)}$

\subsubsection{Stellar Cluster}

The H.E.S.S. group reported Westerlund 2, a young open stellar cluster which contains dozen O-stars and two Wolf-Rayet stars, is an extended gamma-ray source, HESS J1023-575. ${ }^{42)}$ This is a new source class and the gamma-ray emission mechanism is a new challenge to theorists: extended profile suggests there might be collective effects, possibly of stellar winds in the cluster.

\subsubsection{Galactic Center}

The Galactic Center is a confirmed TeV source (by CANGAROO-II, ${ }^{43)}$ Whipple, ${ }^{44)}$ and H.E.S.S. ${ }^{45)}$ ), but the nature of the emission is not known. The energy spectrum shows a simple power-law shape, not compatible 
with dark matter annihilation signal, ${ }^{46)}$ but the position coincides with the central radio source, Sgr A*.47)

Moreover, when the Galactic center source and the nearby source, G0.9+0.1, are subtracted from the H.E.S.S. data, emission extended in galactic longitude for roughly $2^{\circ}$ and also in galactic latitude with a characteristic width of about $0.2^{\circ}$ were seen. ${ }^{48)}$ The powerlaw index of the spectrum (2.3) of this Galactic ridge gamma-ray emission is harder than the local cosmic-ray spectrum (2.7): this may indicate the propagation effects are less pronounced than in the Galaxy as a whole due to the proximity of particle accelerators.

\subsubsection{Unidentified Sources}

Significant number of the $\mathrm{TeV}$ sources distributed near the Galactic plane are unidentified. ${ }^{8)}$ Some have no compelling counterparts, but some are completely dark in other wavelength. ${ }^{49)}$ Former sources could be identified if the angular resolution of Cherenkov telescopes are improved in future detectors, but latter sources bring up a new mystery in astrophysics. Multiwavelength observations of these sources are going on to reveal their identities.

HESS J1908+063 was discovered during the extended H.E.S.S. survey of the Galactic plane, and it coincides with the recently reported MILAGRO unidentified source MGRO J1908+06. ${ }^{50}$ )

TeV J2032+4130 was discovered by HEGRA ${ }^{51)}$ in the Cygnus complex region from their Galactic plane survey, and confirmed by Whipple ${ }^{52)}$ and MAGIC. ${ }^{53)}$ It is also within the extended MILAGRO source MGRO $\mathrm{J} 2031+41 .{ }^{54)}$

Funk et al. studied the $\mathrm{TeV}-\mathrm{GeV}$ connection in Galactic sources comparing the TeV sources with the EGRET catalog. ${ }^{55)}$ Surprisingly, few common sources are found in terms of positional coincidence and spectral consistency. Distribution of integrated energy flux (Fig. 2) shows almost separate peaks for $\mathrm{TeV}$ sources and EGRET sources, which means the current $\mathrm{TeV}$ sensitivity is much better than $\mathrm{GeV}$ sensitivity. The $\mathrm{TeV}$ upper limits put strong constraints on simple power-law extrapolation of several of the EGRET spectra and thus strongly suggest cutoffs in the unexplored energy range from $10 \mathrm{GeV}$ to $100 \mathrm{GeV}$.

\subsection{Extragalactic Sources}

Table IV is a list of known extragalactic sources in the $\mathrm{TeV}$ region in the order of redshift $(z)$.

\subsubsection{Blazars}

Most of sources listed in Table IV are blazar-type active galactic nuclei (AGN), including the second and third established TeV sources, Mrk 421 and Mrk 501. The prominent feature of gamma-ray emission from this class is the rapid time variability, which should be related to the central engine of activity, accretion process around supermassive black holes. In many cases, gammaray emission is accounted by inverse Compton process by high-energy electrons, which naturally explains X-ray and $\mathrm{TeV}$ correlated time variation, but hadronic process is still a viable option. ${ }^{11)}$

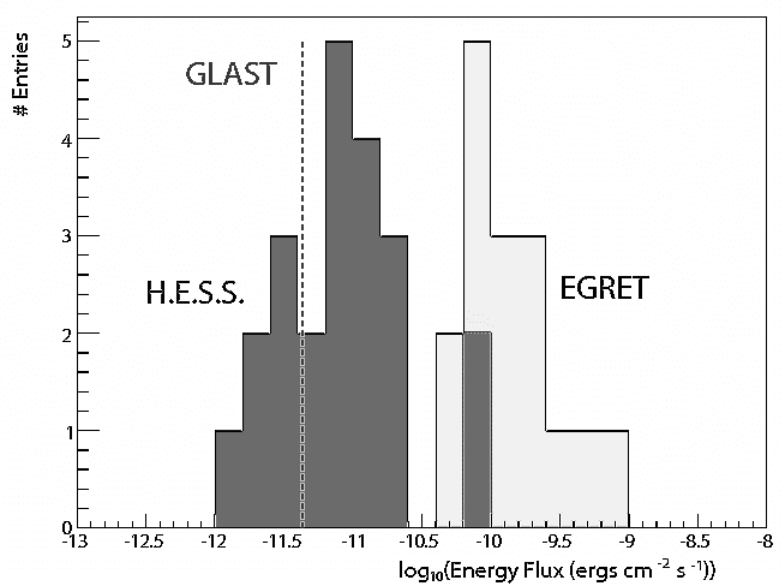

Fig. 2. Distribution of integrated energy flux for sources in the Inner Galaxy. ${ }^{55)}$ For EGRET the energy flux between $1 \mathrm{GeV}$ and $10 \mathrm{GeV}$, for the H.E.S.S. sources, the energy flux between $1 \mathrm{TeV}$ and $10 \mathrm{TeV}$ is shown. Also shown is the sensitivity prediction for the GLAST-LAT for a typical location in the Inner Galaxy $\left(\ell=10^{\circ}, b=0^{\circ}\right)$.

Table IV. Known extragalactic TeV sources as of March 2008. ${ }^{9}$ ) (See Table II for abbreviation of source class.)

\begin{tabular}{|l|c|l|l|}
\hline Object & $z$ & Class & Discovered \\
\hline \hline M87 & 0.004 & Radio & HEGRA, 2003 \\
Mrk 421 & 0.031 & HBL & Whipple, 1992 \\
Mrk 501 & 0.034 & HBL & Whipple, 1996 \\
1ES 2344+514 & 0.044 & HBL & Whipple, 1998 \\
Mrk 180 & 0.046 & HBL & MAGIC, 2006 \\
1ES 1959+650 & 0.047 & HBL & 7TA, 1999 \\
BL Lac & 0.069 & LBL & MAGIC, 2007 \\
PKS 0548-322 & 0.069 & HBL & HESS, 2007 \\
PKS 2005-489 & 0.071 & HBL & HESS, 2005 \\
PKS 2155-304 & 0.116 & HBL & Durham, 1999 \\
H 1426+428 & 0.129 & HBL & Whipple, 2002 \\
1ES 0229+428 & 0.140 & HBL & HESS, 2007 \\
H 2356-309 & 0.165 & HBL & HESS, 2006 \\
1ES 1218+304 & 0.182 & HBL & MAGIC, 2006 \\
1ES 1101-232 & 0.186 & HBL & HESS, 2006 \\
1ES 0347-121 & 0.188 & HBL & HESS, 2007 \\
1ES 1011+496 & 0.212 & HBL & MAGIC, 2007 \\
PG 1553+113 & $>0.25$ & HBL & HESS, 2006 \\
3C279 & 0.536 & FSRQ & MAGIC, 2007 \\
\hline
\end{tabular}

In July 2006, the H.E.S.S. group reported an exceptionally strong flare of PKS 2155-304. ${ }^{56)}$ The peak flux was seven times the flux observed from the Crab nebula, and the flux varied at time scale as short as one minute. ${ }^{57)}$ The CANGAROO-III group confirmed this flaring activity but in a different time zone. ${ }^{58)}$ This short time variability set limit on the size and Doppler factor of gamma-ray emission region near the central massive black hole of the blazar. Assuming the emission region has a size comparable to the Schwarzschild radius of a $\sim 10^{9} M_{\odot}$ black hole, Doppler factors greater than 100 are required to accommodate the observed variability time scales. ${ }^{57)}$

The MAGIC group reported a detection of $\mathbf{3 C 2 7 9},{ }^{59)}$ a prominent flat-spectrum-radio-quasar-type AGN in the $\mathrm{GeV}$ region observed by EGRET ${ }^{60)}$ It was believed that its distance $(z=0.536)$ was too far to be detected 


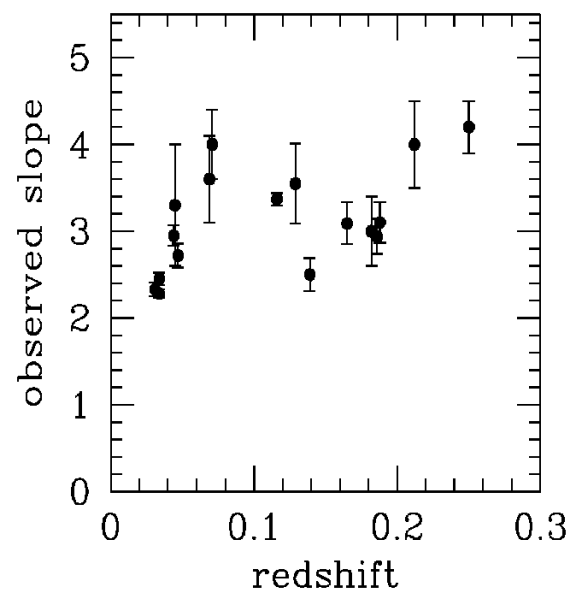

Fig. 3. Observed spectral indices for AGN in the VHE region. ${ }^{3)}$

in very-high-energy gamma-rays due to the absorption effect by the extragalactic infrared background radiation (see next subsection), and we should draw attention to further observations of this object, since the current statistics is too limited to draw spectral information.

\subsubsection{Extragalactic background radiation}

Stecker et al. ${ }^{61)}$ pointed out that extragalactic diffuse infrared background radiation blocks the propagation of $\mathrm{TeV}$ gamma-ray over large distances $(z \gtrsim 0.1$ or $d \gtrsim 100 \mathrm{Mpc}$ ) by producing electron-positron pairs. However, measuring the intensity of the background infrared radiation is a difficult task, since subtracting foreground sources from observed data is a very complicated process. Recent progress has been summarized by many authors: see ref., ${ }^{62)}$ for example. In turn, we can infer the intensity from observation of $\mathrm{TeV}$ spectra of distant sources, assuming the spectra at sources are known: this cannot be known, in fact, so what we can do in practice is to place an upper limit of the intensity of infrared background radiation. Such studies (ref., ${ }^{63)}$ for example) indicate the infrared background radiation might be at lower level than anticipated. This is a result placing constraint on galaxy formation theory, and is a good example of potential power of $\mathrm{TeV}$ gamma-ray astronomy toward cosmology. ${ }^{64)}$

Fig.3 is a plot of spectral indices of AGN in the TeV energy region against redshift. ${ }^{3)}$ If the intrinsic spectra of AGN are similar, there should be a rising tendency in this plot as redshift increases, but we cannot draw clear conclusion from this plot: surely we need more samples.

\subsubsection{M87}

HEGRA reported a detection of $\mathbf{M 8 7}$, a nearby radio galaxy in the Virgo cluster $(z=0.00436$ or $\sim 16 \mathrm{Mpc})$ in 2003. ${ }^{65)}$ H.E.S.S. confirmed the gamma-ray emission of this source, which showed the short-time variability of 2-day scale based on observation between 2003 and 2006. ${ }^{66)}$ This time scale is the order of the light crossing time of the central black hole. The VERITAS group also detected a gamma-ray signal in 2007. ${ }^{67)}$

\subsubsection{Centaurus $A$}

Recent data from the Pierre Auger Observatory suggests this nearby giant radio galaxy $(z=0.0018)$ could be a source of ultra-high-energy cosmic rays. ${ }^{68)}$ If true, one may expect it to emit gamma-rays. ${ }^{69)}$ However, in the $\mathrm{TeV}$ region, only upper limits on gamma-ray flux are reported except one evidence reported in 1970 's. ${ }^{70-72)}$

\subsubsection{Clusters of Galaxies}

As the largest systems in the Universe, clusters of galaxies can harbor high-energy cosmic-rays for cosmological time which may be accelerated in merger shocks and/or accretion shocks, and can emit gamma-rays at detectable level via various possible processes. ${ }^{73)}$

The Whipple group observed Perseus $(z=0.018)$ and Abell $2029(z=0.078)$, but extended gamma-ray emission signals were not observed. ${ }^{74}$ The H.E.S.S. group looked for gamma-ray emission from two nearby clusters, Abell $496(z=0.033)$ and Coma $(z=0.024) .{ }^{75)}$ The CANGAROO-III group selected Abell $4038(z=0.028)$ and Abell $3667(z=0.055)^{76)}$ for target. Again, both groups could not see a hint of signal of gamma-ray emission.

\section{Future Projects}

With these impressive discoveries, next-generation large-scale atmospheric Cherenkov telescope systems are under hot discussion in recent years to enhance the sensitivity in $\mathrm{TeV}$ gamma-rays furthermore. The H.E.S.S. group is constructing a huge $28 \mathrm{~m}$ diameter telescope, called H.E.S.S. II, at the center of the present array, ${ }^{77)}$ and the MAGIC II telescope, an advanced copy of the present MAGIC $17 \mathrm{~m}$ telescope $80 \mathrm{~m}$ apart, will be completed this year in order to enjoy the merit of stereoscopic observation. ${ }^{78)}$ In the longer range, the size of next-generation projects naturally requires international collaboration. CTA (Cherenkov Telescope Array) ${ }^{79)}$ is one of such projects and is lead mainly by H.E.S.S. and MAGIC collaborators. AGIS (Advanced Gammaray Imaging System) ${ }^{80)}$ is another initiative proposed mainly by VERITAS collaborators. These projects aim to increase the sensitivity by a factor of ten, as well as to enhance the gamma-ray energy range both lower and higher regions. The trend seen in the Kifune plot (Fig.4) predicts a bright future of $\mathrm{TeV}$ astronomy with an order of one thousand sources in mid 2010's.

\section{Summary}

Now the very-high-energy window of the Universe is open, and Cherenkov telescopes are providing additional 2-3 decades to the photon spectra of high-energy objects in the sky. We can see wider variety of source classes among seventy $\mathrm{TeV}$ sources than anticipated, indicating cosmic accelerators are ubiquitous in the Universe. However, understanding the underlying physical processes is still at a preliminary stage. Together with GLAST (P. Michelson, in these proceedings.), to be launched in 2008 to observe $\mathrm{GeV}$ gamma-rays from space, we can expect many findings from the exploration of high-energy Universe with next-generation ground-based gamma-ray detectors. 


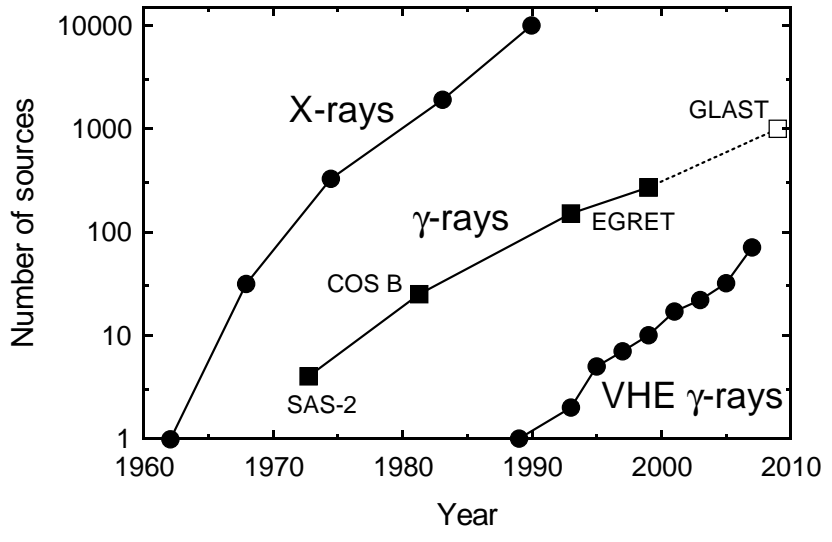

Fig. 4. Yearly increase of X-ray, gamma-ray and very-high-energy (VHE) gamma-ray sources. (This type of plot is called as Kifune plot, named after Prof. Tadashi Kifune who invented it.)

\section{Acknowledgment}

This work is partly supported by Grant-in-Aid for Scientific Research from the Ministry of Education, Culture, Sports, Science and Technology.

1) In this article, very high energy (VHE) means the energies from $100 \mathrm{GeV}$ to $10 \mathrm{TeV}$, and has the same meaning of $\mathrm{TeV}$ region.

2) J. Hinton: Rapporteur talk at 30th Int. Cosmic Ray Conf., Merida, Mexico, 2007; arXiv:0712.3352 (2007).

3) A. De Angelis O. Mansutti, and M. Persic: La Rivista del Nuovo Cimento 31 (2008) 187-246; arXiv:0712.0315 (2007).

4) T.C. Weekes: Very High Energy Gamma-ray Astronomy (Institute of Physics Publishing, Bristol and Philadelphia, 2003).

5) A. M. Hillas: Proc. 19th Int. Cosmic Ray Conf. (La Jolla, USA, 1985) Vol.3, p.445.

6) T.C. Weekes et al.: Astrophys. J. 342 (1989) 379.

7) A. Kohnle et al.: Astroparticle Phys. 5 (1996) 119.

8) F. Aharonian et al.: Science 309 (2005) 746-749; Astrophys. J. 636 (2006) 777.

9) See http://tevcat.uchicago.edu/ or http://www.icrr.utokyo.ac.jp/ ${ }^{\sim}$ morim/TeV-catalog/index.html for more information.

10) http://www.mppmu.mpg.de/ rwagner/sources/

11) F.A. Aharonian: Very High Energy Cosmic Gamma Radiation - A Crucial Window on the Extreme Universe (World Scientific Publishing, Singapore, 2004).

12) H. Muraishi et al.: Astron. Astrophys., 354, (2000) L57.

13) R. Enomoto et al.: Nature 416 (2002) 823.

14) F. Aharonian et al.: Nature 432 (2004) 75.

15) F.A. Aharonian et al.: Astron. Astrophys. 464 (2007) 235.

16) H. Katagiri et al.: Astrophys. J. 619 (2005) L163.

17) F. Aharonian et al.: Astron. Astrophys. 437 (2005) L7.

18) S. Hoppe et al.: arXiv:0709.4103 (2007).

19) F. Aharonian et al.: Astron. Astrophys. 370 (2001) 112.

20) J. Albert et al.: Astron. Astrophys. 474 (2007) 937.

21) F. Aharonian et al:: arXiv:0803.0702 (2008).

22) B. Katz and E. Waxman: JCAP 01 (2008) 018 and references therein.

23) E.G. Berezhko and H.J. Völk: arXiv:0707.4647 (2007).

24) D. Ellison, D. J. Patnaude, P. Slane, P. Blasi, and S. Gabici: Astrophys. J. 661 (2007) 879.

25) Y. Uchiyama, F.A. Aharonian, T. Tanaka, T. Takahashi, and Y. Maeda: Nature 449 (2007) 576

26) Y. Butt, T. Porter, B. Katz, and E. Waxman: arXiv:0801.4954
(2007).

27) J. Albert et al.: Astrophys. J. 664 (2007) L87.

28) T.B. Humensky et al.: arXiv:0709.4298 (2007).

29) F. Aharonian et al:: arXiv:0801.3555 (2008).

30) F. Aharonian et al:: Astron. Astrophys. 469 (2007) L1.

31) See, for example, V. Tatischeff: arXiv:0804.1004 (2008) for a recent review.

32) GLAT Collaboration: S. Funk: arXiv:0709.3127 (2007).

33) F. Aharonian et al.: Astron. Astrophys. 460 (2006) 365.

34) E.V. Gotthelf, J.P. Halpern, F. Camilo, C. Markwardt, and J. Swank: The Astronomer's Telegram \#1392 (2008); L. Kuiper, W. Hermsen, M. Klein-Walt, and R. Wijands: The Astronomer's Telegram \#1405 (2008).

35) J.G. Kirk, L. Ball and O. Skjaeraasen: Astropart. Phys. 10 (1999) 31.

36) F. Aharonian et al.: Astron. Astrophys. 442 (2005) 1.

37) A. Kawachi et al.: Astrophys. J. 607 (2004) 949.

38) F. Aharonian et al.: Astron. Astrophys. 460,(2006) 743.

39) J. Albert et al.: Science 312 (2006) 1771.

40) G. Maier et al.: arXiv:0709.3661 (2007).

41) J. Albert et al.: Astrophys. J. 665 (2007) L51.

42) F. Aharonian et al.: Astron. Astrophys. 467, (2007) 1075.

43) K. Tsuchiya et al.: Astrophys. J. 606 (2004) L115.

44) K. Kosack et al.: Astropys. J. 608 (2004)L97.

45) F. Aharonian et al.: Astron. Astrophys. 425 (2004) L13.

46) F. Aharonian et al.: Phys. Rev. Lett. 97 (2006) 221102.

47) C. van Eldik et al.: arXiv:0709.3729 (2007).

48) F. Aharonian et al.: Nature 439 (2006) 695.

49) F. Aharonian et al.: Astron. Astrophys. 477 (2008) 353.

50) A. Djannati-Atai et al.: arXiv:0710.2418 (2007).

51) F.A. Aharonian et al.: Astron. Astrophys. 393 (2002) L37.

52) M.J. Lang et al.: Astron. Astrophys. 423 (2004) 415; A. Konopelko et al.: Astrophys. J. 658 (2007) 1062.

53) J. Albert et al.: Astrophys. J. 675 (2008) L25.

54) A.A. Abdo et al.: Astrophys. J. 664 (2007) L91.

55) S. Funk, O. Reimer, D.F. Torres and J.A. Hinton: arXiv:0710.1584 (2007).

56) W. Benbow, L. Costamante and B. Giebels: The Astronomer's Telegram \#867, July 27, 2006.

57) F. Aharonian et al.: Astrophys. J. 664 (2007) L71.

58) Y. Sakamoto et al.: Astrophys. J. 676 (2008) 113.

59) M. Teshima et al.: arXiv:0709.1475 (2007).

60) R.C. Hartman et al.: Astrophys. J. 558 (2001) 583 and references therein.

61) F.W. Stecker, O.C. de Jager, and M.H. Salamon: Astrophys. J. 390 (1992) L49.

62) M. Raue and D. Mazin: arXiv:0802/0129 (2008).

63) F. Aharonian et al.: Nature 440 (2006) 1018.

64) See, for example, F.W. Stecker: arXiv:0709.0904 (2007).

65) F. Aharonian et al.: Astron. Astrophys. 403 (2003) L1.

66) F. Aharonian et al.: Science 314 (2006) 1424.

67) P. Colin et al: arXiv:0709.3663 (2007).

68) The Pierre Auger Collaboration: Science 318 (2007) 938.

69) N. Gupta: arXiv:0804.3017 (2008); M. Kachelriess, S. Ostapchenko, and R. Tomas: arXiv:0805.2608 (2008)

70) J.E. Grindlay, H.F. Helmken, R.H. Brown, J. Davis, and L.R. Allen: Astrophys. J. 197 (1975) L9.

71) F. Aharonian et al.: Astron. Astrophys. 441 (2005) 465.

72) S. Kabuki et al.: Astrophys. J. 668 (2007) 968 and references therein.

73) S. Inoue, F.A. Aharonian and N. Sugiyama: Astrophys. J. 628 (2005) L9 and references therein.

74) J.S. Perkns et al.: Astrophys. J. 644 (2006) 148.

75) W. Domainko et al.: arXiv:0710.4057 (2007).

76) R. Kiuchi et al.: Proc. 30th Int. Cosmic Ray Conf. (Merida, Mexico, 2007), paper 166.

77) P. Vincent et al.: Proc. 29th Int. Cosmic Ray Conf. (Pune, India, 2005), Vol. 5, 163.

78) F. Goebel et al.: arXiv:0709.2605 (2007).

79) http://www.cta-observatory.org/

80) http://gamma1.astro.ucla.edu/agis/index.php/Main_Page 